\title{
ETHNIC DIFFERENCES IN HSV1 AND HSV 2 SEROPREVALENCE in Amsterdam, the Netherlands
}

\author{
MA Kramer (mkramer@ggd.amsterdam.nl)1, DG Uitenbroek ${ }^{1}$, JK Ujcic-Voortman ${ }^{1}$, C Pfrommer ${ }^{1}$, J Spaargaren², \\ R A Coutinho ${ }^{3,4}$, NHTM Dukers-Muijrers ${ }^{5}$ \\ 1. Health Service of Amsterdam, Amsterdam, the Netherlands \\ 2. Laboratory of Infectious Diseases, Groningen, the Netherlands \\ 3. Center for Infectious Disease Control, National Institute for Public Health and the Environment, Bilthoven, the Netherlands \\ 4. Academic Medical Centre, University of Amsterdam, Amsterdam, the Netherlands \\ 5. South Limburg Health Service, Geleen, the Netherlands
}

\begin{abstract}
Herpes simplex virus type 1 (HSV1) and 2 (HSV2) infection can lead to significant morbidity, and HSV2 is considered a risk factor for HIV transmission. The majority of HSV-infected people are asymptomatic and unaware of their infection. We aimed to determine the HSV1 and HSV2 prevalence among various ethnic groups in a large urban area in the Netherlands. In 2004, serum samples from a populationbased serum repository of 1,325 people over 18 years living in Amsterdam were tested for HSV1 and HSV2 antibodies in order to determine high-risk groups. Prevalence ratios were estimated and all analyses were weighted by sex, age, and ethnicity. In the general population of Amsterdam, $67 \%$ had HSV1 antibodies, $22 \%$ had HSV2 antibodies, $15 \%$ had HSV1 and HSV2 antibodies, and $26 \%$ had no indication of HSV infection. In multivariate analyses, HSV1 seroprevalence increased with age, and was higher among people of Turkish and Moroccan origin, homosexual men, and individuals with low educational level. HSV2 seroprevalence was associated with increasing age, Surinamese/Antillean background, and having a history of sexually transmitted infections (STI). These differences between ethnic groups in Amsterdam regarding the distribution of HSV1 and HSV2 infection emphasise the importance of an ethnicspecific approach of serological testing as well as campaigns aimed at behavioural change and counselling to raise awareness of the risk of HSV transmission.
\end{abstract}

\section{Introduction}

Herpes simplex virus (HSV) causes oral-facial, genital and cutaneous infections. Both people with symptomatic lesions and asymptomatic individuals can shed virus particles particles and transmit HSV. When transmitted vertically, HSV causes neonatal herpes, which can lead to neurological damage or death [1]. In addition, the increase in genital transmission of herpes simplex virus type 1 (HSV1) and the evolving evidence that genital HSV infection is a potent facilitator of the sexual transmission of human immunodeficiency virus (HIV) are a considerable public health concern [2-5].

The majority of HSV-infected individuals are asymptomatic and unaware of their infection. Sero-epidemiological studies suggest that serologic testing for genital herpes identifies more infected individuals than are recognised clinically $[1,6]$. Therefore, control strategies at the population level will not be fully effective if limited to symptom management.

Two types of HSV can be distinguished, type 1 (HSV1) and 2 (HSV2). HSV1 is a common childhood infection, with prevalence ranging from $52-84 \%$ in Europe [7]. HSV2 is mostly sexually transmitted and is more restricted to subgroups of the population, such as men who have sex with men (MSM), with prevalence ranging from $4-80 \%$ [8]. Population-based studies on HSV in the Netherlands are scarce and studies have been conducted primarily in high-risk groups, such as attendees of sexually transmitted infections (STI) clinics and MSM [9,10]. Two studies conducted in the general population found HSV1 seroprevalences of $60 \%$ and $70 \%$ and HSV2 seroprevalences of $8 \%$ and $35 \%[7,11]$. In the first study, non-native Dutch individuals were underrepresented and the second study excluded men.

HSV vaccine studies have reached phase II; phase III efficacy trials are in progress. Since vaccine studies on HSV2 are progressing, the epidemiology of HSV has received increasing attention [12]. By testing samples from the first population-based serum repository in Amsterdam, we aimed to obtain a representative picture of the HSV1 and HSV2 prevalence among men and women of different origin and background in a large urban area. Almost half the residents of Amsterdam are non-Dutch and originate mainly from Suriname and the Antilles (22\%), Morocco (18\%), and Turkey (11\%) [13].

\section{Methods}

\section{Study population and sampling procedure}

The population-based serum repository is a result of the Amsterdam Health Monitor (AHM) which was carried out from April until June 2004 [14]. With approval of the Medical Ethical Committee of the Amsterdam Medical Centre, the AHM collected information about the health of the general population in Amsterdam to gain more insight into the determinants for public health in Amsterdam. The study sample was drawn from the Amsterdam municipal registers in five city districts. These districts comprised a population representative to the Amsterdam general population in terms of ethnic mix, sex, and age. The sample was stratified by ethnic origin and five age groups (18-34, 35-44, 45-54, 55-64 and 65 years or older). Within each stratum a random sample was drawn with an oversampling of Turkish and Moroccan people who are relatively underrepresented in the total population and had a lower participation rate in previous national and local surveys in the Netherlands. Ethnic origin was classified by the country of birth of the participant and/or the country of birth of the parents. Ethnicity was determined as foreign if the participant or one of their parents was born outside the Netherlands. 
The total study sample consisted of 4,042 residents aged 18 years and older. The participants were invited by mail to a face-toface interview based on a structured questionnaire regarding issues such as socio-demographics, chronic and infectious diseases, drug use, and living environment. For questions on sexual behaviour, participants could choose between interview-administration and self-administration of the answers.

The final response rate on the AHM was 44\% [14]. After written informed consent, $79 \%$ of the participants $(n=1,376)$ donated blood for the serum repository. There was a relation between whether a person responded or not and their age, sex and ethnicity. Among those who did respond, there were no significant differences in age and sex between participants who gave blood and participants who only participated in the survey, but those who did not give blood were more often of Turkish origin.

When comparing the participants who agreed to have a blood sample taken and the control group of those who did not respond to the survey at all, certain subgroups were more willing than others to give blood: Those who gave blood were more often $45-64$ years-old (45\% versus $36 \%$ ) and of Dutch ethnic origin (33\% versus $23 \%$ ), whereas the youngest and oldest age groups (16\% versus $24 \%$ of the $18-34$ year-olds, and $17 \%$ versus $20 \%$ of those 65 years-old and older), the male participants ( $46 \%$ versus $51 \%$ ), and those of Moroccan ethnic origin ( $21 \%$ versus $26 \%$ ) were less likely to agree to give blood.

\section{Serological testing}

Of the 1,376 blood samples, 1,362 were tested for HSV1 and HSV2 using HSV1 and HSV2 antibody assays (HerpesSelect, Focus Technologies, USA). According to the manufacturer instructions, test results below a cut-off value of 0.9 were defined as HSV1- or HSV2-negative, values between 0.9 and 1.1 as equivocal, and test results over 1.1 as HSV1- or HSV2-positive. Samples with an equivocal HSV test result $(n=29 ; 1.6 \%$ for HSV1 and $0.5 \%$ for HSV2,), and samples for which data on age or country of birth $(n=8)$ was not available were excluded, resulting in a final dataset of 1,325 participants for statistical analysis.

\section{Variables and statistical analysis}

For respondents of Turkish and Moroccan ethnic origin (age 18-34 years) we defined their generation as: 1) first generation: participant and one or both parents born in a foreign country; 2) second generation: participant born in the Netherlands and one or both parents born in a foreign country. For males, attraction to mainly the same sex was defined as homosexual preference.

Relative risks were estimated instead of odds ratios, because the rare event assumption was not reached. Prevalence ratios were estimated using a modified Poisson regression model [15]. To correctly estimate the standard error for the estimated relative risk, a robust standard error was obtained by implementing a repeated statement in the SAS GENMOD procedure. Variables with a $\mathrm{p}<0.10$ in univariate analyses were entered into the multivariate model into which ethnicity, age and sexual preference were forced. Interaction between risk factors and gender was explored, but no significant interaction was found to be present.

The questionnaire also included detailed questions on sexual behaviour. In total, 976 (74\%) of the 1,325 respondents who gave blood answered these questions. People of Turkish and Moroccan origin and those with a low educational level were more likely to decline answering questions on their sexual behaviour. Univariate analyses were carried out to examine the association between seropositivity for HSV1 or HSV2 and sexual behaviour in the past year (e.g. sexual contact or not, number of partners, anal sex).

Defining results above a cut-off value of 1.1 as positive for HSV2 in populations with multiple infections (e.g. African populations) has yielded a high rate of false positive results in earlier studies $[16,17]$. In those studies, false positives were less frequently detected in sera with HSV index values of over 3.5 compared to values in the low positive range (1.1-3.5). As suggested in one of the studies [16], we conducted sensitivity analyses using a cut-off value of 3.5 (excluding those with an index value between 0.9 and 3.5). The risk factors for HSV infection that were found in these analyses were comparable to those obtained in analyses with a cut-off value of 1.1 (data not shown).

All the analyses were weighted according to the cell weighting method [18] by sex in two groups, age in five groups, and ethnicity in six groups, to account for oversampling and deviations of the sample distribution in sex, age, and ethnicity from the general population aged 18 years and older in Amsterdam in 2004. In this way results were representative for the adult population in Amsterdam [14].

\section{Results}

\section{Characteristics of the study sample}

The study population (those who participated in the survey and gave a blood sample) consisted of 611 men and 714 women. The median age for men was 51 years (interquartile range (IQR) 41-62 years) and for women 47 years (IQR 37-58 years). The majority of men $(80 \%)$ and women $(76 \%)$ in the sample had a Dutch, Turkish, or Moroccan ethnic background, and $40 \%$ of the participants had a medium educational level (lower vocational school or secondary school). More women (20\%) than men (17\%) had had an HIV test, but more men $(11 \%)$ than women $(8 \%)$ reported a history of one or more STIs.

\section{Prevalence of HSV infection}

Overall, $67 \%$ (95\% confidence interval (Cl): 63.5-71.0) of the population had HSV1 antibodies, $22 \%(95 \% \mathrm{Cl}$ : 18.9-24.9) were positive for HSV2, and $15 \%(95 \% \mathrm{Cl}: 12.1-17.2)$ were co-infected with HSV1 and HSV2.

Twenty-six percent $(95 \% \mathrm{Cl}: 21.9-29.2)$ of the population had no serological evidence of HSV infection. Most of those were of Dutch $(33 \%)$ or other Western (26\%) ethnic origin. As shown in Figure 1, the proportion of people with no HSV infection decreased with age, from $37 \%$ in the group of $18-34$ year-olds to $11 \%$ in the 55-64 year-olds. This age effect was not visible in people of Turkish, Moroccan and other non-Western origin. The proportion of seronegative people in these populations was very low (around $5 \%$ ) across all the age groups.

\section{Determinants and predictors of HSV1 infection}

Over $80 \%$ of the people with Turkish, Moroccan, and other non-Western ethnicity were HSV1 seropositive across all the age groups (Figure 2). In contrast, the HSV1 seroprevalence of those originating from the Netherlands and other Western countries increased with age: from $40 \%$ in the age group $18-34$ years to $80 \%$ in the age group 65 years and older. Ethnic background therefore had the largest influence in the youngest age group of the 18-34 year-olds who were twice as likely to be infected if they were of Turkish, Moroccan, and other non-Western origin. Responsible for 
the high seroprevalence among people from Morocco and Turkey were primarily immigrants of the first generation. In the youngest age group (18-34 years), we observed a non-significant tendency (exact chi-square test, $p=0.07$ ) towards higher seroprevalence in immigrants of the first generation among whom 98\% (78/80) were HSV1 seropositive compared to $87 \%(20 / 23)$ of the second generation.

In multivariate analyses, HSV1 seropositivity was associated with increasing age, being of Turkish or Moroccan ethnicity, and low educational level (Table). After controlling for ethnicity, age and education, homosexual men were more likely to be seropositive for HSV1 than women and heterosexual men. No association was found between HSV1 seroprevalence and sexual behaviour in the past year.

\section{Determinants and predictors of HSV2 infection}

People of Surinamese, Antillean, and other non-Western ethnicity had the highest seroprevalence of HSV2 (26\% and 32\%) compared to people of indigenous Dutch ethnicity (20\%). No significant differences were found between generations of migrants, but older individuals and those who had ever had an STI were more likely to be HSV2 seropositive (Figure 3 and Table). None of the estimated risks for HSV2 seropositivity was significantly different for males and females, but HSV2 seroprevalence was (not significantly) higher in females and in homosexual men compared to heterosexual men.

Additional analyses on the association between sexual behaviour and HSV2 infection were done for the part of the study population which had responded to those questions $(n=976)$. These analyses showed that individuals with more than one sexual partner in the past year had a 1.56 higher risk of HSV2 infection $(95 \% \mathrm{Cl} 1.13$ 2.16) compared to those with one or no sexual partner in the past year.

\section{Discussion and conclusion}

Our study demonstrates clear differences in HSV distribution among ethnic groups in Amsterdam, the Netherlands. The HSV1 seroprevalence among migrants born outside the Netherlands reflects the prevalence reported in their country of origin, because most HSV1 acquisition occurs during childhood or young adulthood $[8,19,20]$. As a corollary, a lower HSV1 seroprevalence was found among the second generation of migrants, who were born in the Netherlands. This could partly be explained by improved socioeconomic conditions and adaptation to certain western cultural habits. The HSV1 seroprevalence among indigenous Dutch persons in Amsterdam (59\%) supports the results of a European serological survey in 1994 , in which $57 \%$ of the Dutch samples were found to be HSV1-positive [7]. Consistent with population-based studies worldwide, we found an association between HSV1 infection and age as well as socio-economic factors such as low education $[7,8,20,21]$.

HSV2 prevalence likewise varied with geographic origin and this might explain the higher HSV2 seroprevalence in Amsterdam in 2004 (22\%) compared to the prevalence in the general population in the Netherlands in 1994 (9\%), since our study included nonindigenous individuals. In addition, a higher concentration of high risk profiles in urban areas, and an increase in sexual risk behaviour over time might explain the discrepancy.

In line with other studies, HSV2 prevalence in our study was highest among people originating from Suriname, the Antilles and other non-Western countries (excluding Morocco and Turkey)
$[6,8,11,21,22]$. High HSV2 seroprevalence in these populations living in Amsterdam reflects parameters of sexual behaviour such as a higher number of sexual partners and concurrent partnerships among men originating from Suriname, the Netherlands Antilles, and Africa [22,23].

As HSV2 infection facilitates HIV transmission, it is worth noting that the largest non-Dutch group of HIV-infected individuals in the Netherlands are sub-Saharan Africans (44\%), followed by people

\section{F I G U R E}

Weighted prevalence of HSV seronegativity by age and ethnicity among the general population of Amsterdam in 2004

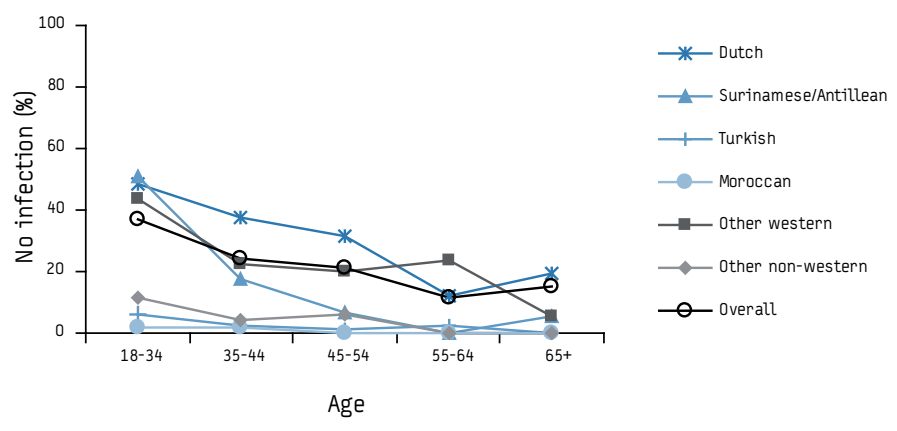

F I G U R E 2

Weighted HSV1 prevalence by age and ethnicity among the general adult population of Amsterdam in 2004

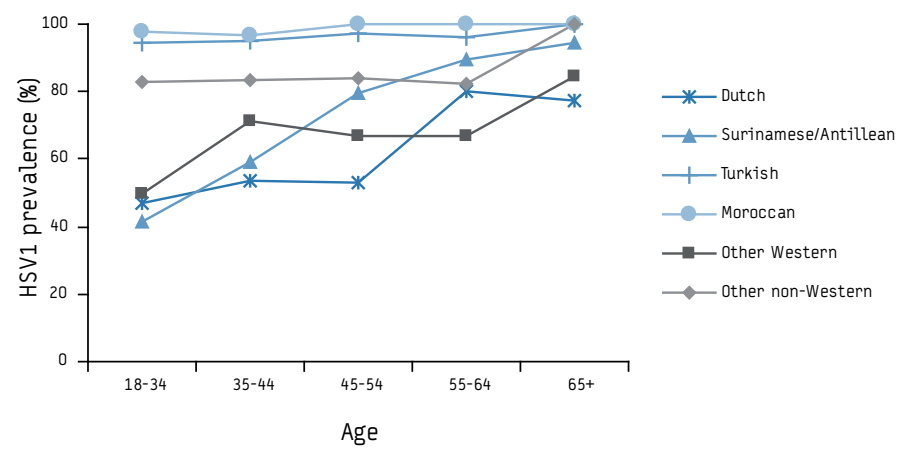

F I G U R E 3

Weighted HSV2 prevalence by age and ethnicity among the general adult population of Amsterdam in 2004

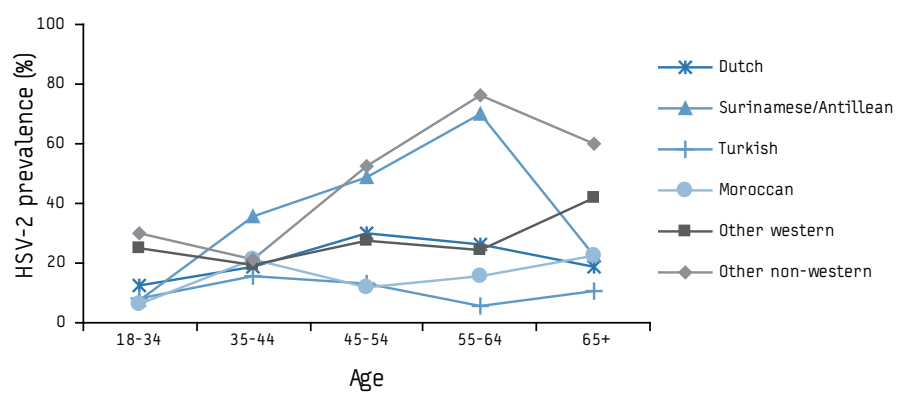


T A B L E

Multivariate model of associations with HSV1 infection among the general population of Amsterdam, $2004(\mathrm{n}=1,325)$

\begin{tabular}{|c|c|c|c|c|c|}
\hline & \multirow[b]{2}{*}{ N } & \multicolumn{2}{|c|}{ HSV1 } & \multicolumn{2}{|c|}{ HSV2 } \\
\hline & & Rate+SE (95\% CI) & PRR (95\% CI) & Rate+SE (95\% CI) & PRR (95\% CI) \\
\hline Age (per 10 years) & 1,325 & & $1.09(1.06-1.15)^{\star \star \star}$ & & $1.26(1.14-1.39)^{\star \star \star}$ \\
\hline $\begin{array}{l}\text { Ethnicity } \\
\text { Dutch } \\
\text { Surinamese/Antillean } \\
\text { Turkish } \\
\text { Moroccan } \\
\text { Other Western } \\
\text { Other non-Western } \\
\text { a }\end{array}$ & $\begin{array}{l}435 \\
88 \\
314 \\
276 \\
136 \\
76\end{array}$ & $\begin{array}{l}58.9+2.7(53.6-64.2) \\
63.1+7.6(48.1-78.2) \\
95.2+1.7(91.9-98.5) \\
98.3+1.1(96.2-99.9) \\
64.2+6.0(52.3-76.0) \\
85.1+4.6(75.9-94.4)\end{array}$ & $\begin{array}{l}1.00^{\star \star \star} \\
1.15(0.90-1.47) \\
1.73(1.51-1.98) \\
1.78(1.56-2.03) \\
1.10(0.91-1.33) \\
1.59(1.36-1.87)\end{array}$ & $\begin{array}{l}19.9+2.1(15.9-23.9) \\
25.7+5.3(17.1-34.3) \\
11.1+2.3(6.6-15.5) \\
12.4+2.3(7.8-16.9) \\
27.2+5.3(16.6-37.7) \\
32.0+5.6(20.8-43.2)\end{array}$ & $\begin{array}{l}1.00^{\star \star} \\
1.60(1.03-2.51) \\
0.80(0.49-1.31) \\
0.81(0.51-1.29) \\
1.42(0.93-2.18) \\
2.01(1.34-3.02)\end{array}$ \\
\hline $\begin{array}{l}\text { Educational level } \\
\text { High } \\
\text { Medium } \\
\text { Low }\end{array}$ & $\begin{array}{l}268 \\
530 \\
308\end{array}$ & $\begin{array}{l}55.4+3.9(47.8-62.9) \\
71.5+2.8(66.0-77.0) \\
88.1+2.1(83.9-92.3)\end{array}$ & $\begin{array}{l}1.00^{\star \star} \\
1.22(1.05-1.41) \\
1.19(1.01-1.39)\end{array}$ & $\begin{array}{l}22.0+2.9(16.4-27.7) \\
20.9+2.1(16.8-25.0) \\
24.2+3.5(17.4-31.1)\end{array}$ & \\
\hline History of STI & 125 & $71.9+5.2(61.6-82.4)$ & & $42.6+5.5(31.6-53.6)$ & $1.82(1.30-2.56)^{\star *}$ \\
\hline $\begin{array}{l}\text { Sexual preference } \\
\text { Men-Homosexual } \\
\text { Men-Heterosexual } \\
\text { Women }\end{array}$ & $\begin{array}{l}59 \\
536 \\
691\end{array}$ & $\begin{array}{l}82.1+6.8(68.3-95.8) \\
63.5+3.2(57.1-69.9) \\
68.1+2.5(63.1-73.0)\end{array}$ & $\begin{array}{l}1.36(1.12-1.64) \\
1.00^{\star} \\
1.08(0.96-1.22)\end{array}$ & $\begin{array}{l}29.5+6.5(16.4-42.6) \\
18.2+2.2(13.9-22.6) \\
24.4+2.1(20.3-28.4)\end{array}$ & $\begin{array}{l}1.48(0.74-2.94) \\
1.00 \\
1.27(0.95-1.72)\end{array}$ \\
\hline
\end{tabular}

$\mathrm{SE}=$ standard error; $\mathrm{PRR}=$ prevalence rate ratio

a other Western (Europe, North America, Oceania), other non-Western (Asia, Africa, South America)

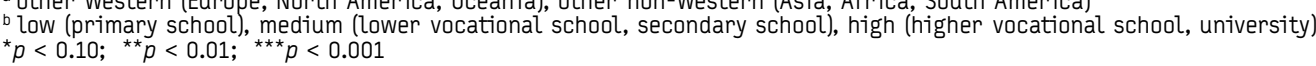

from the Caribbean and Latin America (14\%), predominantly Suriname and the Netherlands Antilles [24]. The HSV2 prevalence we found among people of Moroccan origin corresponds to the prevalence rate (12\%) found in a national HIV sentinel surveillance conducted in Morocco [20]. In contrast, the HSV2 seroprevalence among the Turkish residents of Amsterdam (11\%) differed considerably from the prevalence (42\%) found among pregnant women attending an antenatal clinic in Turkey [19]. It has to be noted, however, that the two groups do not necessarily compare and HSV2 prevalence reported in women are usually higher than in men [6-8].

Consistent with other studies, we observed an increased risk of HSV2 with increasing age across all ethnic groups $[6,7,8,20]$, but also a peak in HSV2 seroprevalence in the age group of 55-64 years old. This probably reflects the increasing number of years of sexual activity. Across geographic areas, attendees of STI clinic consistently had higher prevalence of HSV2 infection [25]. Our study showed that a history of STI and the number of sexual partners were markers of higher risk of HSV2 infection.

Unlike other studies conducted in the US, Africa and Europe, we found no association between gender and HSV seropositivity [6-8]. However, we did find evidence of a higher HSV1 prevalence in homosexual men compared to heterosexual men and women, and the data may suggest that HSV2 prevalence was higher in both women and homosexual men compared to heterosexual men.

Several studies have shown discrepant results as to the interaction between HSV1 and HSV2 [25,26], and it has been proposed that prior HSV1 infection may protect against acquiring HSV2. However, we observed no association between being positive for HSV1 and being negative for HSV2.

The results of the study could have been biased due to the low response rate of $44 \%$. Although this response rate is comparable with several national surveys in the Netherlands (50\%), it is nevertheless a cause for concern. Response rates in Dutch survey research generally tend to be lower compared to similar American and European studies (60-80\%) [27,28]. Two reasons are often mentioned. Firstly, the Netherlands is a highly urbanised country and response rates tend to be lower in urban areas. Secondly, lower response rates could in part be due to higher mobility of the population, especially in Amsterdam. It should also be noted, however, that HSV does not in itself 'cause' non-response. The association between HSV and response is indirect and will have had a relatively modest influence.

For estimation of the prevalences, the data were weighted by sex, age, and ethnicity, both overall and within the subgroups where relevant. The effects of differences in response between groups are addressed in this way and we believe that prevalence from this perspective can be considered representative for the whole adult population of Amsterdam.

Limitations of the HerpesSelect antibody assay are well known. Discrepant results are mainly obtained in populations with multiple infections. Use of a higher cut-off value ( $>3.5)$ instead of a cut-off value of $>1.1$ have been recommended by other studies. A higher positive index value resulted in increased specificity (98\%), but has shown to reduce sensitivity $(90 \%)[16,29]$. In our study, risk factors identified with a cut-off value of 3.5 were comparable to those identified when using 1.1 as cut-off value.

Current HSV control strategies include behavioural intervention and symptom management. However, a combined approach of behavioural change, suppressive therapy and serological testing for genital herpes simplex for those with current or recent STI or high-risk behaviour, is more likely to have a substantial impact on the prevention of HSV acquisition and transmission. Serological testing gives the opportunity to counsel patients. Patients who have been counselled on the natural history of HSV and the correlation between HSV transmission and HIV infection may be more aware of the fact that they have an increased risk of acquiring HIV or other STIs and of what they can do to limit this risk [30]. 
It is possible to limit the spread of HSV1, as shown by the somewhat lower prevalence of HSV1 in the second generation compared to the first generation of Turkish and Moroccan migrants. However, since HSV1 infections are primarily acquired during childhood and first generation immigrants are more likely to become infected before taking up residence in the Netherlands, a further decline in HSV1 transmission also depends on the prevention efforts in their countries of origin.

A decrease in HSV1 seroprevalence among migrants of the first generation would lead to a larger group of young people susceptible to HSV1 primary infection, for example genital infection. On the other hand, a decrease in seroprevalence would at the same time mean more people who could potentially benefit from an HSV2 vaccine, which currently has been shown to be effective only in HSV1-negative women [3].

Our results provided useful insight into the distribution of HSV in a large urban area with a high proportion of residents of non-Western origin. Our study showed that migrant groups have different patterns and epidemiology for infectious diseases and these patterns could differ between generations. These findings emphasise the importance of an ethnic-specific approach to raise awareness for the prevention of HSV transmission.

\section{Acknowledgements}

The serum repository was supported by a grant from the Health Service of Amsterdam Research and Development Fund, the Netherlands. The Amsterdam Health Monitor was supported by a grant from the Municipal of Amsterdam and a grant from the Centre for Prevention and Health Services Research, National Institute for Public Health and the Environment, the Netherlands.

The authors would like to thank Ronald Geskus for the realisation of this project; the participants for their willingness to cooperate; the technicians of the Public Health Laboratory for preparing and storing the serum samples; and Lucy Philips for editing the final manuscript.

\section{References}

1. Brugha R, Keersmaekers K, Renton A, Meheus A.. Genital herpes infection: a review. Int J Epidemiol. 1997;26(4):698-709.

2. Wald A, Link K. Risk of human immunodeficiency virus infection in herpes simplex virus type 2-seropositive persons: a meta analysis. J Infect Dis. 2002;185(1):45-52.

3. Cowan FM, Copas A, Johnson AM, Ashley R, Corey L, Mindel A. Herpes simplex type 1 infection: a sexually transmitted infection of adolescence? Sex Transm Infect. 2002;78(5):346-8.

4. Haddow LJ, Dave B, Mindel A, McPhie KA, Chung C, Marks C, et al. Increase in rates of herpes simplex virus type 1 as a cause of anogenital herpes in western Sydney, Australia, between 1979 and 2003. Sex Transm Infect. 2006;82(3):255-9.

5. Manavi K, ,McMillan A, Ogilvie M. Herpes simplex virus type 1 remains the principal cause of initial anogenital herpes in Edinburgh, Scotland. Sex Transm Dis. 2004;31(5):322-4.

6. Wald A. HSV-2 transmission: risk factors and virus shedding. Herpes. 2004;11 Suppl 3:130A-137A.

7. Pebody RG, Andrews N, Brown D, Gopal R, De Melker H, François G, et al. The seroepidemiology of herpes simplex virus type 1 and 2 in Europe. Sex Transm Infect. 2004;80(3):185-91.

8. Smith JS, Robinson NJ. Age-specific prevalence of infection with herpes simplex virus types 2 and 1: a global review. J Infect Dis. 2002;186 Suppl $1: 53-28$

9. Roest RW, van den Meijden WI, van Dijk G, Mulder PG, Verjans GM, Osterhaus ADME. Prevalence and association between herpes simplex virus types 1 and 2-specific antibodies in attendees at a sexually transmitted disease clinic. Int J Epidemiol. 2001;30(3):580-8.
10. Dukers NH, Bruisten SM, van den Hoek JA, de Wit JB, van Doornum GJ, Coutinho RA. Strong decline in herpes simplex virus antibodies over time among youn homosexual men is associated with changing sexual behavior. Am J Epidemiol. 2000;152(7):666-73.

11. Gaytant MA, Steegers EA, van Laere M, Semmekrot BA, Groen J, Weel JF, et at Seroprevalences of herpes simplex virus type 1 and type 2 among pregnant women in the Netherlands. Sex Transm Dis. 2002;29(11):710-4.

12. Stanberry LR, Spruance SL, Cunningham AL, Bernstein DI, Mindel A, Sacks S, et al. Glycoprotein-D-adjuvant vaccine to prevent genital herpes. $N$ Engl J Med. 2002;347(21):1652-61.

13. Available from the website of the Department for Research and Statistics, Municipal of Amsterdam, the Netherlands: http://www.os.amsterdam.nl

14. Agyemang C, Ujcic-Voortman J, Uitenbroek D, Foets M, Droomers M. Prevalence and management of hypertension among Turkish, Moroccan and native Dutch ethnic groups in Amsterdam, the Netherlands: the Amsterdam Health Monitor Survey. J Hypertens. 2006;24(11):2169-76.

15. Zou G. A modified poisson regression approach to prospective studies with binary data. Am J Epidemiol. 2004;159(7):702-6.

16. Ashley-Morrow R, Nollkamper J, Robinson NJ, et al. Performance of focus ELISA tests for herpes simplex virus type (HSV-1) and HSV-2 antibodies among women in ten diverse geographical locations. Clin Microbiol Infect. 2004 Jun;10(6):530-6.

17. Hogrefe W, Su X, Song J, Ashley R, Kong L. Detection of herpes simplex virus-2 immunoglobulin $G$ antibodies in African sera by using recombinant $g G 2$, Western blotting, and gG2 inhibition. J Clin Microbiol. 2002;40(10):3635-40.

18. Kalton G, Flores-Cervantes I. Weighting Methods. J Off Stat. 2003;19:81-97.

19. Arseven G, Tuncel E, Tuncel S, Sönmez E, Gülen AK. Distribution of HSV1 and HSV2 antibodies in pregnant women. Mikrobiyol Bul. 1992;26(4):359-66. [In Turkish ]

20. Cowan FM, French RS, Mayaud P, Gopal R, Robinson NJ, de Oliveira SA, Seroepidemiological study of herpes simplex virus types 1 and 2 in Brazil, Estonia, India, Morocco, and Sri Lanka. Sex Transm Infect. 2003;79(4):286-90.

21. Schillinger JA, Xu F, Sternberg MR, Armstrong GL, Lee FK, Nahmias AJ, et al. National seroprevalence and trends in herpes simplex virus type 1 in the United States, 1976-1994. Sex Transm Dis. 2004 Dec;31(12):753-60.

22. Weiss H. Epidemiology of herpes simplex virus type 2 infection in the developing world. Herpes 2004; 11(Suppl1):24A-35A.

23. Gras MJ, van Benthem BH, Coutinho RA, van den Hoek A. Determinants of highrisk sexual behavior among immigrant groups in Amsterdam: implication for interventions. J Acquir Immune Defic Syndr. 2001;28(2):166-72.

24. de Boer IM, Op de Coul EL, Koedijk FD, van Veen MG, van Sighem AI, van de Laar MJ. HIV and sexually transmitted infections in the Netherlands in 2005. Bilthoven: National Institute for Public Health and the Environment; 2006. Report no.: 441100024. Available from: http://rivm.nl/bibliotheek/ rapporten/441100024.html

25. Cowan FM, Johnson AM, Ashley R, Corey L, Mindel A. Antibody to herpes simplex virus type 2 as a serological marker of sexual lifestyle in populations. BMJ. 1994;309(6965):1325-9

26. Looker KJ, Garnett GP. A systematic review of the epidemiology and interaction of herpes simplex virus types 1 and 2. Sex Transm Infect. 2005;81(2):103-7.

27. Aromaa A, Koponen P, Tafforeau J, Vermeire C; HIS/HES Core Group.. Evaluation of health interview surveys and health examination surveys in the European Union. Eur J Public Health. 2003:13(3 Suppl):67-72.

28. Van Loon AJ, Tijhuis M, Picavet HS, Surtees PG, Ormel J. Survey non-response in the Netherlands: effects on prevalence estimates and associations. Ann Epidemiol. 2003;13(2):105-10.

29. Golden MR, Ashley-Morrow R, Swenson P, Hogrefe WR, Handsfield HH, Wald A. Herpes simplex virus type 2 (HSV-2) Western blot confirmatory testing among men testing positive for HSV-2 using the Focus enzyme-linked immunosorbent assay in a sexually transmitted disease clinic. Sex Transm Dis. 2005;32(12):771-7.

30. Patel R. Educational interventions and the prevention of herpes simplex virus transmission. Herpes. 2004;11 Suppl 3:155A-160A.

This article was published on 12 June 2008

Citation style for this article: Kramer M, Uitenbroek D, Ujcic-Voortman J, Pfrommer C, Spaargaren J, Coutinho RA, Dukers-Muijrers N. Ethnic differences in HSV1 and HSV seroprevalence in Amsterdam, the Netherlands. Euro Surveill. 2008:13(24):pii=18904 Available online: http://www.eurosurveillance.org/ViewArticle.aspx?ArticleId=18904 